\title{
Vacuum energy and the latent heat of AdS-Kerr black holes
}

\author{
Brian P. Dolan* \\ Department of Mathematics, Heriot-Watt University, Colin Maclaurin Building, \\ Riccarton, Edinburgh, EH14 4AS, United Kingdom \\ and Maxwell Institute for Mathematical Sciences, \\ Edinburgh, United Kingdom \\ (Received 18 August 2014; published 2 October 2014)
}

\begin{abstract}
Phase transitions for rotating asymptotically anti-de Sitter black holes in four dimensions are described in the $P-T$ plane, in terms of the Hawking temperature and the pressure provided by the cosmological constant. The difference between constant angular momentum and constant angular velocity is highlighted; the former has a second order phase transition while the latter does not. If the angular momentum is fixed there a line of first order phase transitions terminating at a critical point with a second order phase transition and vanishing latent heat, while if the angular velocity is fixed there is a line of first order phase transitions terminating at a critical point with infinite latent heat. For constant angular velocity the analytic form of the phase boundary is determined, latent heats derived and the Clapeyron equation verified.
\end{abstract}

DOI: $10.1103 /$ PhysRevD.90.084002

PACS numbers: 04.60.-m, 04.70.Dy

\section{INTRODUCTION}

There has recently been interest in interpreting the cosmological constant in an asymptotically de Sitter or anti-de Sitter (AdS) black hole space-time to be a pressure in a thermodynamic sense. The thermodynamically conjugate variable would then be interpreted as a volume associated to the black hole, though it would not necessarily be related to any notion of geometric volume [1-3]. The idea of varying the cosmological constant goes back to [4,5], but the interpretation adopted here was first given in [1]. In this paper we reexamine some of the known thermodynamics of asymptotically AdS-Kerr black holes from this perspective, emphasizing the role that the pressure plays in the analysis.

As usual in thermodynamics, the phase structure depends on the constraints. For example when the angular momentum $J$ is held fixed there is a critical point with a second order phase transition at finite pressure and temperature, first found in [6]. This constant $J$ phase transition is known to have mean field exponents $[7,8]$, putting it in the same universality class as a van der Waals gas and the phase diagram (Fig. 3) looks the same as that of a van der Waals gas.

On the other hand fixing the angular velocity $\Omega$ results in a phase diagram which, like the one-dimensional Ising model, has no second order transition at any finite temperature. There is a critical point at finite $T$ and $P$ where the free energy has a cusp, but the latent heat diverges there. Strictly speaking there is a second critical point, with vanishing latent heat, but it is at infinite $T$ and there is no phase transition as one cannot pass through this point- this is similar to the 1-d Ising model, though there the critical point is at $T=0$.

\footnotetext{
*B.P.Dolan@hw.ac.uk
}

The phase structure for constant $\Omega$ and constant $J$ is different in the pressure-temperature plane. ${ }^{1}$ In the former case the phase boundary is determined by the condition $\rho=2 P$, where $\rho=\frac{M}{V}$ is the black hole mass per unit volume, while it is not so easy to find an analytic expression for constant $J$. Many familiar notions from ordinary thermodynamics are applicable, such as the Clapeyron equation for the slope of the phase boundary in the $P-T$ plane, but there are also significant differences.

While the analysis produces some explicit expressions for phase boundary curves in the $P-T$ plane and latent heats in black hole phase transitions that have not appeared in the literature before, these are not the main point of the paper, being just trivial consequences of the structure of the Hawking-Page phase transitions. Rather the main point is to emphasize the shift in viewpoint that occurs when the thermodynamic volume is introduced. The phase diagram in Fig. 2 is the same as that of [11], but drawn using thermodynamic variables rather than the geometric variables that are explicit in the metric. This is done to emphasize the physics of the thermodynamics: the free energy is a single valued function of the geometric variables, but is multiple valued in terms of thermodynamic variables, giving the different branches in the $P-T$ plane that are the hallmark of phase transitions in the grand canonical ensemble. This conceptual shift may well prove to be important for the analysis of rotating superfluids in the AdS/CFT correspondence [12].

In Sec. II, static nonrotating black holes are treated as a warm-up for the constant $\Omega$ case in Sec. III and constant $J$ in Sec. IV. Conclusions are given in Sec. V.

\footnotetext{
${ }^{1}$ The fixed $J$ and fixed $\Omega$ ensembles were analyzed in [9] and [10] in terms Ehrenfest equations.
} 


\section{STATIC BLACK HOLES}

A nonrotating, neutral, asymptotically anti-de Sitter black hole has line element

$$
d^{2} s=-f(r) d t^{2}+f^{-1}(r) d r^{2}+r^{2} d \Omega^{2},
$$

with

$$
f(r)=1-\frac{2 m}{r}-\frac{\Lambda}{3} r^{2},
$$

and $d \Omega^{2}=d \theta^{2}+\sin ^{2} \theta d \phi^{2}$. The horizon radius, $r_{h}$, is determined by the largest real root of $f(r)=0$ giving

$$
m=\frac{r_{h}}{2}\left(1-\frac{\Lambda}{3} r_{h}^{2}\right),
$$

which is the asymptotically $\operatorname{AdS}$ equivalent of the Arnowitt-Deser-Misner (ADM) mass, $M=m$, in the nonrotating case. Following [1], $M$ will be identified with the enthalpy,

$$
H(S, P)=\frac{1}{2}\left(\frac{S}{\pi}\right)^{\frac{1}{2}}\left(1+\frac{8 P S}{3}\right)
$$

where the Bekenstein-Hawking entropy is $1 / 4$ of the event horizon area

$$
S=\pi r_{h}^{2}
$$

and $P=-\frac{\Lambda}{8 \pi}$ is the pressure ( $G_{N}$ and $\hbar$ are set to 1$)$. The temperature follows either from the surface gravity

$$
T=\frac{f^{\prime}\left(r_{h}\right)}{4 \pi}=\frac{\left(1-\Lambda r_{h}^{2}\right)}{4 \pi r_{h}}
$$

using (1) or from the thermodynamic relation

$$
T=\left.\frac{\partial H}{\partial S}\right|_{P}=\frac{(1+8 P S)}{4 \sqrt{\pi S}}
$$

using (3); these are the same formulas written in geometric and thermodynamic variables, respectively.

The temperature has a minimum at $S=\frac{1}{8 P}$ when

$$
T_{\min }=\sqrt{\frac{2 P}{\pi}}
$$

For $S<\frac{1}{8 P}$ the heat capacity

$$
C_{P}=\left.T \frac{\partial S}{\partial T}\right|_{P}=2 S\left(\frac{8 P S+1}{8 P S-1}\right)
$$

is negative while for $S>\frac{1}{8 P}$ it is positive and it diverges at $T_{\min }$.
The thermodynamic volume is the Legendre transform of the pressure [1,2], namely

$$
V=\frac{\partial M}{\partial P}=\frac{4}{3 \sqrt{\pi}} S(T, P)^{\frac{3}{2}}=\frac{4 \pi}{3} r_{h}^{3} .
$$

This is a rather surprising result as there is no a priori reason for the thermodynamic volume to be related to a geometric volume. ${ }^{2}$ Indeed when rotation is introduced there is no such obvious relation [3].

The Gibbs free energy is the Legendre transform of the enthalpy,

$$
\begin{aligned}
G(T, P) & =H-T S=\frac{1}{4} \sqrt{\frac{S(T, P)}{\pi}}\left(1-\frac{8 P S(T, P)}{3}\right) \\
& =\frac{r_{h}}{4}\left(1+\frac{\Lambda}{3} r_{h}^{2}\right)
\end{aligned}
$$

with

$$
S(T, P)=\frac{\pi T^{2}-P \pm T \sqrt{\pi^{2} T^{2}-2 \pi P}}{8 P^{2}}
$$

(the heat capacity is positive for the plus sign, negative for the minus sign and diverges when $P \rightarrow \pi T^{2} / 2$ ).

We have the thermodynamic relation

$$
d G=-S d T+V d P
$$

and, for AdS space-time, $G=0$ and so $S_{\mathrm{AdS}}=V_{\mathrm{AdS}}=0$ (this is the thermodynamic volume of AdS space-time, not a geometric volume). For a black hole, on the other hand, the thermodynamic volume is given by (8).

If $P S>\frac{3}{8}$ the Gibbs free energy of the black hole is negative and thus lower than that of anti-de Sitter spacetime, the former is then the more stable thermodynamic configuration, while for $P S<\frac{3}{8}$ pure anti-de Sitter spacetime is the more stable and any black hole with $S<\frac{3}{8 P}$ will tend to evaporate. This is the Hawking-Page phase transition [14], which occurs on the line $\Lambda r_{h}^{2}=-3$, or

$$
S=\frac{3}{8 P} \Rightarrow P=\frac{3 \pi}{8} T^{2},
$$

when the two states can exist together as shown in the $P-T$ plane in Fig. 1 below. (Similar phase diagram plots appeared in [15] and are shown here with a view to

\footnotetext{
${ }^{2}$ It is not immediately clear how the geometric volume of a black hole might be defined, as $r$ is a timelike coordinate and $t$ a spacelike coordinate for $r<r_{h}$. Inside the event horizon surfaces of constant $t$ have a time-dependent metric. Note that the entropy and the volume are not independent for a Schwarzschild black hole. This is not a pathology: they are independent for rotating black holes and the above formula can be obtained by taking the nonrotating limit of the rotating case [13].
} 

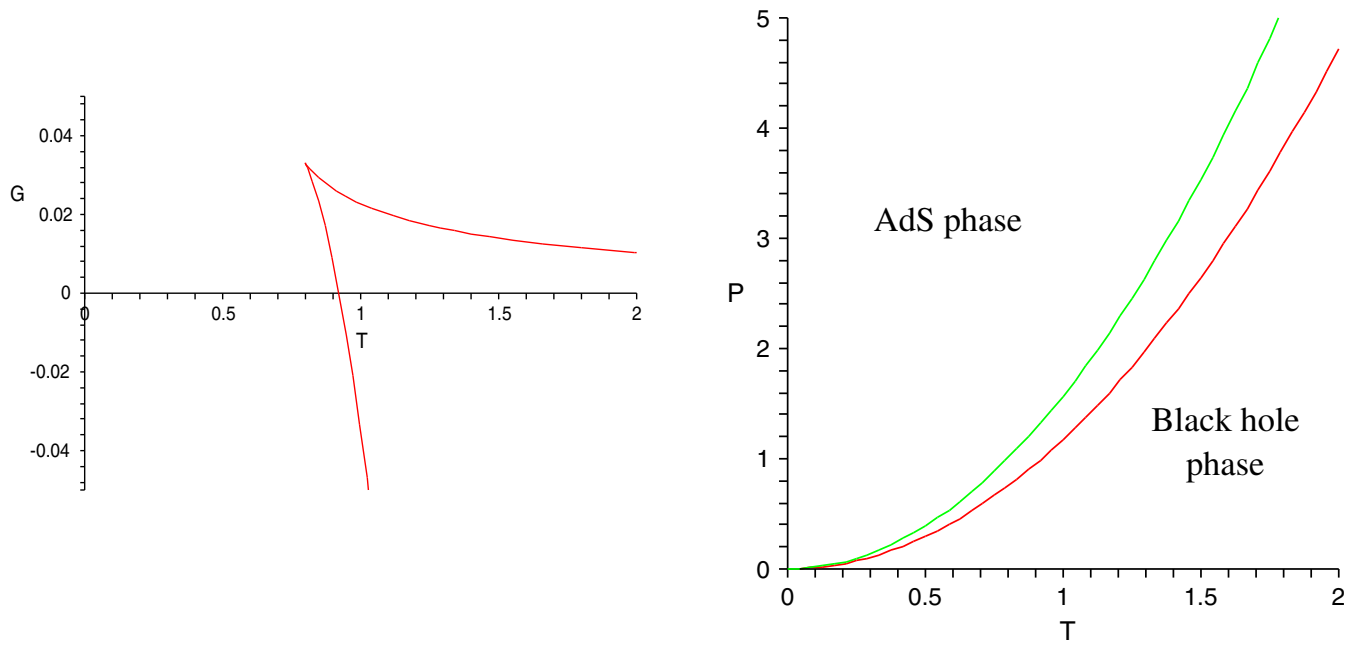

FIG. 1 (color online). Left: The black hole free energy, for static black holes, at fixed $P=1$. The heat capacity is negative on the upper branch, positive on the lower branch and diverges at the cusp. The Hawking-Page temperature is where $G=0$. Right: The coexistence curve of the Hawking-Page phase transition is the lower (red) line; the heat capacity diverges on the upper (green) line. The lower branch of the free energy tends to minus infinity on the $P=0$ axis.

extending the analysis to constant angular velocity in the next section.)

Clearly there is a jump in entropy,

$$
\Delta S=\frac{3}{8 P}
$$

when a black hole nucleates from pure AdS space time, energy must be supplied to form the black hole at constant temperature and the latent heat is

$$
L=T \Delta S .
$$

From the form of the coexistence curve in (11) the latent heat is

$$
L=\frac{1}{\pi T}=\sqrt{\frac{3}{8 \pi P}}
$$

which is equal to the mass on the coexistence curve, in the black hole phase. The latent heat is nonzero for any finite $T$ and goes to zero as $T \rightarrow \infty$, as though the system were aiming for a second order phase transition but could not reach it. For asymptotically flat space-times, $P=0$ and the latent heat is infinite; hence black holes will not spontaneously nucleate in Minkowski space.

The Clapeyron equation for static black holes follows from equating the Gibbs free energy of the two phases at a point on the coexistence curve. For AdS space-time $G_{\mathrm{AdS}}=0$, so in particular

$$
d G_{\text {AdS }}=0
$$

on the coexistence curve, while for the black hole

$$
d G_{\mathrm{BH}}=-S_{\mathrm{BH}} d T+V_{\mathrm{BH}} d P .
$$

The coexistence curve is defined by $G_{\mathrm{AdS}}=G_{\mathrm{BH}}$, so

$$
\begin{aligned}
0 & =d G_{\mathrm{AdS}}-d G_{\mathrm{BH}}=S_{\mathrm{BH}} d T-V_{\mathrm{BH}} d P \\
\Rightarrow \frac{d P}{d T} & =\frac{S_{\mathrm{BH}}}{V_{\mathrm{BH}}}=\frac{3}{4 r_{h}} .
\end{aligned}
$$

Since there is no black hole in pure AdS space-time we define

$$
\Delta V=V_{\mathrm{BH}}, \quad \Delta S=S_{\mathrm{BH}}
$$

giving

$$
\frac{d P}{d T}=\frac{\Delta S}{\Delta V},
$$

which is the Clapeyron equation [16]. It is easily checked, using (4) and (11) directly, that indeed $\frac{d P}{d T}=\frac{3}{4 r_{h}}$. The Clapeyron equation for static charged black holes, and its relation to Ehrenfest's equations, was considered in [10].

\section{ROTATING BLACK HOLES}

In this section the analysis of the Hawking-Page phase transition is extended to rotating black holes in asymptotically AdS space times. The ADM mass for a black hole rotating with angular momentum $J$ can be expressed as a function of the entropy, the angular momentum and the pressure [6]

$$
H(S, P, J):=\frac{1}{2} \sqrt{\frac{\left(1+\frac{8 P S}{3}\right)\left(S^{2}\left(1+\frac{8 P S}{3}\right)+4 \pi^{2} J^{2}\right)}{\pi S}},
$$

which is again interpreted here as the enthalpy. 
In asymptotically AdS space-times there can be rotating black holes that are in equilibrium with thermal radiation rotating at infinity $[11,14]$. The black hole is stable against decay if its Euclidean action is less than that of pure AdS, which is zero, so it is stable if the Euclidean action is negative. The Euclidean action of the black hole, $I_{E}$, is a function of $T$, $P$ and the angular velocity, $\Omega$, and is related to the Legendre transform of the ADM mass [17], $I_{E}=\Xi / T$ with

$$
\Xi(T, \Omega, P)=M-S T-J \Omega .
$$

The Legendre transforms can be performed to obtain $\Xi$ explicitly. First express the temperature and the angular velocity as functions of entropy and angular momentum

$$
\begin{gathered}
T=\left.\frac{\partial H}{\partial S}\right|_{J, P}=\frac{1}{8 \pi H}\left[\left(1+\frac{8 P S}{3}\right)(1+8 P S)-4 \pi^{2}\left(\frac{J}{S}\right)^{2}\right] \\
\Omega=\left.\frac{\partial M}{\partial J}\right|_{S, P}=2 \pi^{3 / 2} J \sqrt{\frac{(3+8 P S)}{S\left(3 S^{2}+8 P S^{3}+12 \pi^{2} J^{2}\right)}} .
\end{gathered}
$$

While it is easy to invert the latter to write $J$ as a function of $\Omega$, expressing $S$ explicitly as a function of $T$ requires the solution of an eighth order polynomial equation. Nevertheless we can eliminate $J$ in favor of $\Omega$,

$T(S, \Omega, P)=\frac{\left(64 P^{2} S^{2} \pi-24 P S^{2} \Omega^{2}+32 \pi P S-6 \Omega^{2} S+3 \pi\right)}{4 \pi \sqrt{S(3+8 P S)\left(3 \pi+8 \pi P S-3 \Omega^{2} S\right)}}$.

Solving for $S$ now only involves a quartic, but simpler is to use (21) in (18) to give

$\Xi(T(S, \Omega, P), \Omega, P)=\frac{\sqrt{S}\left(9 \pi+24 P S^{2} \Omega^{2}-64 P^{2} S^{2} \pi\right)}{12 \pi \sqrt{(3+8 P S)\left(3 \pi+8 \pi P S-3 \Omega^{2} S\right)}}$,

which, together with (21), gives $\Xi(T, \Omega, P)$ parametrically in terms of $S$.

The Hawking-Page phase transition is determined by the locus of points where $\Xi=0$, i.e.

$$
S^{2}=\frac{9 \pi}{8 P\left(8 \pi P-3 \Omega^{2}\right)} .
$$

Note that

$$
\Omega^{2} \leq \frac{8 \pi P}{3}
$$

is a condition that must be imposed on $\Omega$ in order to ensure that the Einstein universe at infinity is not rotating faster than the speed of light [11].
The free energy at a fixed pressure $P>\frac{3 \Omega^{2}}{8 \pi}$ is plotted as a function of temperature in Fig. 2, using the dimensionless variables

$$
p=\frac{8 \pi P}{\Omega^{2}} \geq 3, \quad t=\frac{T}{\Omega} \quad \text { and } \quad s=\frac{\Omega^{2} S}{\pi} .
$$

For $p>3$ there are two branches and the Hawking-Page temperature is determined by the point where the lower branch cuts the $t$ axis. As $p \rightarrow 3$ from above the lower branch becomes steeper until it disappears at $p=3$, the upper branch remaining and terminating at $T=\frac{\Omega}{2 \pi}$, where $\Xi=\frac{1}{4 \Omega}$. For $p<3$ there are black hole solutions for all positive $T$ with the black hole free energy $\Xi_{\mathrm{BH}}$ a positive function decreasing monotonically with $T$.

Substituting (23) in (21) gives an analytic expression for the coexistence curve $T(P)$ at fixed $\Omega$ : it has the parametric form

$$
\begin{aligned}
& p=\frac{3\left(s+\sqrt{s^{2}+4}\right)}{2 s}, \\
& t=\frac{\sqrt{2+\sqrt{s^{2}+4}}}{2 \pi \sqrt{s}}
\end{aligned}
$$

and is plotted as the upper-left boundary of the region marked "Black hole phase" in the right-hand panel of Fig. 2. It terminates at $p=3$ and the temperature of the black hole cannot go below $T=\frac{\Omega}{2 \pi}$ for $p \geq 3$. Black holes are stable against decay to AdS in the region labeled "Black hole phase." In this region $\Xi_{\mathrm{BH}}$ is double valued, with a positive and a negative branch, the negative branch being the more stable of the two and also more stable than AdS which has $\Xi_{\mathrm{BH}}=0$. Below the horizontal line $p=3$ the negative branch of $\Xi_{\mathrm{BH}}$ disappears and only the positive branch remains. Across this line the negative branch jumps discontinuously from minus infinity (black hole) to zero $(\mathrm{AdS}){ }^{3}$

When there is rotation present angular momentum contributes to the change in enthalpy across the coexistence curve, which is the latent heat. For the Hawking-Page transition the latent heat is just the mass of the black hole:

(i) On the first order line, $\Xi_{\mathrm{BH}}=M-T S-\Omega J=0$, hence

$$
L=M=T S+\Omega J .
$$

Since the entropy and angular momentum vanish on the AdS side of the transition the jumps in entropy and angular momentum are $\Delta S=S$ and $\Delta J=J$, giving latent heat

\footnotetext{
${ }^{3}$ For finite jumps such phase transitions have been termed zeroth order and it has been suggested that they could occur in superconductors and superfluids [18]. Indeed it was proposed in [11] that the horizontal line $p=3$ may be associated with a superfluid transition.
} 

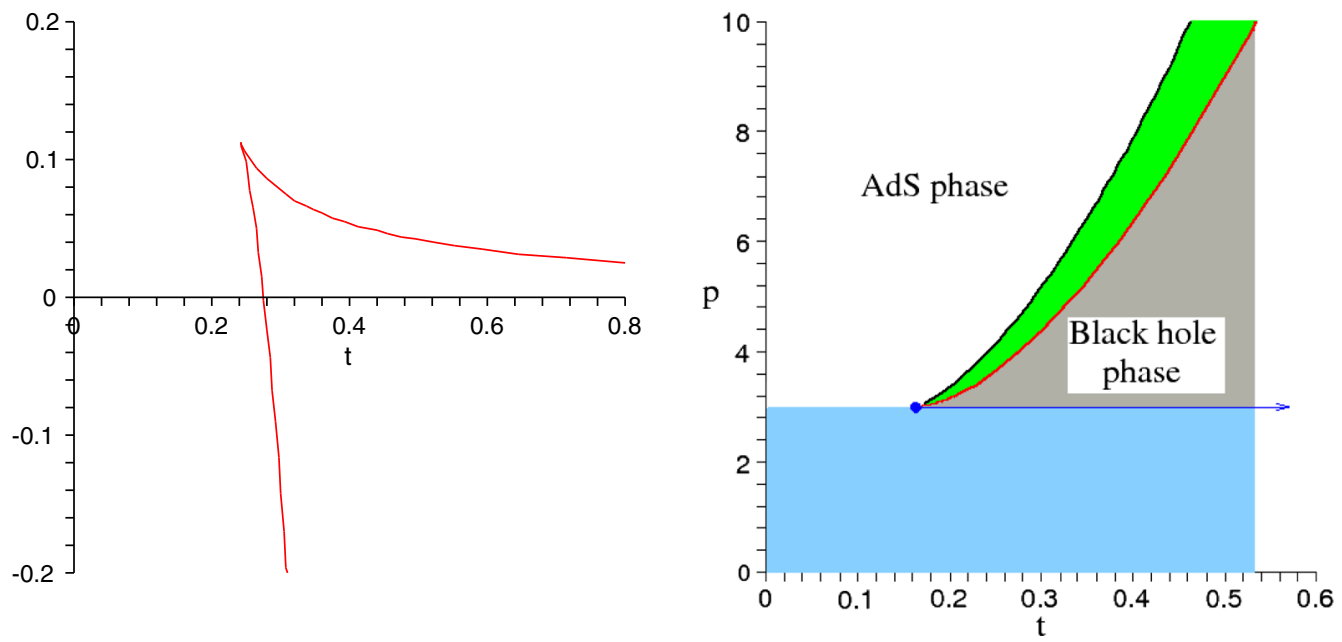

FIG. 2 (color online). Left: The dimensionless free energy for a rotating black hole as a function of the dimensionless variable $t$. The upper branch represents small black holes (negative heat capacity), the lower branch large black holes (positive heat capacity). Only negative $\Xi$ black holes are stable against Hawking-Page decay. The vertical axis is the dimensionless combination $\Omega \Xi$ and the figure is drawn for $p=4$ (other values of $p>3$ move the position of the cusp but the shape of the figure is the same). Right: Phase diagram in the $p-t$ plane. The constraint that the Einstein universe at infinity is not rotating faster than the speed of light imposes the condition $p \geq 3$. In the region below this line $\Xi_{\mathrm{BH}}$ is single valued and positive. In the white region (upper left labeled "AdS phase") there are no black hole solutions (a black hole in this region would have negative entropy). The right-hand boundary of the white region is the locus of points on which the heat capacity diverges (black line), to the right of this there is a wedge shaped region (dark gray, green) in which $\Xi_{\mathrm{BH}}$ has two branches and is positive on both branches. AdS is still the preferred phase in this region as $0=\Xi_{\mathrm{AdH}}<\Xi_{\mathrm{BH}}$. In the gray region, labeled "Black hole phase," one branch of $\Xi_{\mathrm{BH}}$ becomes negative and black holes are more stable than AdS. The Hawking-Page phase transition occurs on the upper-left boundary of the region marked "Black hole phase," where the lower branch of $\Xi_{\mathrm{BH}}$ equals zero.

$$
L=T \Delta S+\Omega \Delta J=\frac{16 \pi^{3} T^{3}}{\left(4 \pi^{2} T^{2}-\Omega^{2}\right)^{2}},
$$

which diverges at the critical point ${ }^{4} T=\frac{\Omega}{2 \pi}, P=\frac{3 \Omega^{2}}{2 \pi}$.

Of course $M=T S+\Omega J$ is not a general formula; it only holds on the coexistence curve and gives a quick and convenient way of determining the curve. It can be combined with the Smarr relation,

$$
M=2(T S+\Omega J-P V),
$$

to give $M=2 P V$, or

$$
\rho=2 P
$$

with $\rho=\frac{M}{V}$, on the coexistence curve.

The Clapeyron equation can be checked using thermodynamic volume, $V=\left.\frac{\partial H}{\partial P}\right|_{S, J}$, first calculated in [3]. Written as a function of $\Omega$ it reads

$$
V=\frac{2 S^{\frac{3}{2}}\left(16 \pi P S-3 \Omega^{2} S+6 \pi\right)}{3 \pi \sqrt{(3+8 P S)\left(3 \pi+8 \pi P S-3 \Omega^{2} S\right)}} .
$$

On the coexistence curve (23) this is

\footnotetext{
${ }^{4}$ This is a critical point in the original sense of the phrase; it is necessary to tune both $T$ and $P$ very carefully to access this point. A critical point in this sense is a separate concept to the notion of zero latent and a "second order" phase transition.
}

$$
V=\frac{2 \pi}{3 \Omega^{3}} \sqrt{s^{3}\left(2+\sqrt{s^{2}+4}\right)} .
$$

Again, as the thermodynamic volume of AdS without a black hole vanishes, $\Delta V=V$ and

$$
\frac{\Delta S}{\Delta V}=\frac{3 \Omega}{2 \sqrt{s\left(2+\sqrt{s^{2}+4}\right)}},
$$

and this is indeed equal to $\frac{d P}{d T}$ along the coexistence curve, from (26).

Another way of writing this is to use (28) to give

$$
\frac{d P}{d T}=\frac{S}{V}=\frac{2 P S}{M}
$$

hence, at a given value of $\Lambda$, the slope of the coexistence curve is determined by the entropy per unit mass of the black hole.

(ii) The Clapeyron equation does not hold across the horizontal zeroth order transition line in Fig. 2, because $\Xi_{\mathrm{BH}} \neq 0$ there. In fact $\Xi_{\mathrm{BH}}<0$ on this line and the free energy jumps across it.

The free energy $\Xi_{\mathrm{BH}}$ is plotted in Fig. 2 as a function of the dimensionless variable $t$ in (25), with $p=4$. There is a cusp at the minimum temperature $t=\frac{1}{2 \pi}$, where the heat capacity and the latent heat diverge, but no phase transition. 


\section{THE CALDARELLI, COGNOLA AND KLEMM PHASE TRANSITION}

It was found in [6] that there is a phase transition for asymptotically AdS rotating black holes with fixed angular momentum, $J$, between small and large black holes, the Caldarelli-Cognola-Klemm (CCK) phase transition. The phase structure associated with constant $J$ transitions has been more extensively studied from the point of view of varying $P$ than the constant $\Omega$ case [15]. It is more like the familiar liquid-gas transition than the constant $\Omega$ case and in higher dimensions there can be a triple point associated with three different black hole phases [15]. There is no Hawking-Page phase transition for constant $J$, because AdS with no black hole cannot have nonzero $J$. The results in this section are not new and are included for completeness and comparison to the constant $\Omega$ case in Sec. III.

The thermodynamic form of the mass for the asymptotically AdS-Kerr metric is (17), the temperature is (19) and the thermodynamic volume (29) is, in terms of $J$,

$$
V=\left.\frac{\partial M}{\partial P}\right|_{S, J}=\frac{2}{3 \pi M}\left\{S^{2}\left(1+\frac{8 P S}{3}\right)+2 \pi^{2} J^{2}\right\} .
$$

This is greater than (or equal to when $J=0$ ) the naïve geometric result $V=\frac{4 \pi}{3}\left(\frac{S}{\pi}\right)^{\frac{3}{2}}$, as first observed in [3].

In the $P-V$ plane the CCK transition mimics the van der Waals gas-liquid phase transition very closely. Below a critical temperature $T_{c}$ the transition is first order, culminating at a second order transition at $T_{c}$, in the same universality class as the van der Waals transition (with mean field exponents [7,8]) and no transition for $T>T_{c}$. The free energy

$$
G(T, P)=M(S, P)-S T
$$
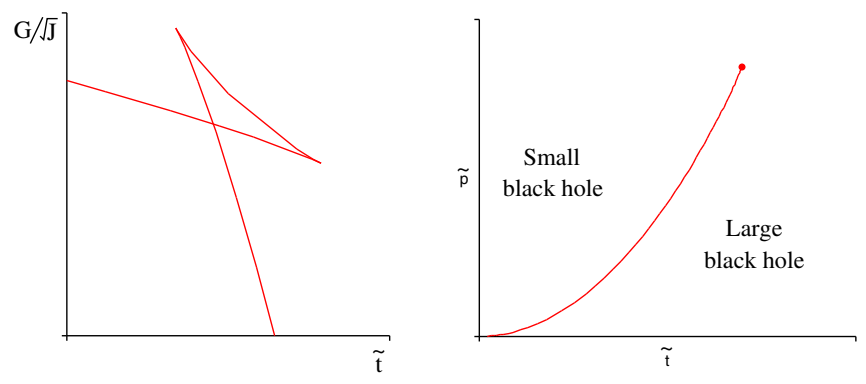

FIG. 3 (color online). Left: Gibbs free energy, $G / \sqrt{J}$, as a function of $\tilde{t}$ for a black hole at constant $J$ with $\tilde{p}=<\tilde{p}_{c}$. Right: Coexistence curve at fixed $J$, in the $\tilde{p}-\tilde{t}$ plane. The curve ends at the critical point $\tilde{t}_{c}=0.0417, \tilde{p}_{c}=0.144$.

is plotted in Fig. 3, as a function of $T$ at fixed $P$, together with the coexistence curve in the $P-T$ plane.

Just like the van der Waals equation of state there is a line of second order phase transitions, but here between large and small black holes, terminating at critical point where there is a second order phase transition.

The heat capacity at constant $J$ and $P$ is

$$
C_{J, P}=\left.T \frac{\partial S}{\partial T}\right|_{J, P}
$$

The full expression is not very illuminating and we shall focus on the spinodal curve at constant $J$, the locus of points in the $S-P$ plane where the heat capacity diverges. In terms of the dimensionless variables

$$
\tilde{p}=16 \pi P J, \quad \tilde{s}=\frac{S}{2 \pi J}
$$

the spinodal curve is given by a quartic polynomial in $\tilde{p}$,

$$
\tilde{p}^{4} \tilde{s}^{8}+4 \tilde{s}^{5}\left(2 \tilde{s}^{2}+3\right) \tilde{p}^{3}+18 \tilde{s}^{4}\left(\tilde{s}^{2}+5\right) \tilde{p}^{2}+36 \tilde{s}\left(6 \tilde{s}^{2}+1\right) \tilde{p}+162 \tilde{s}^{2}+81-27 \tilde{s}^{4}=0 .
$$

Defining a dimensionless temperature $\tilde{t}=T \sqrt{J}$, curves of constant $\tilde{p}$ are plotted in the $\tilde{s}-\tilde{t}$ plane in Fig. 4.

The spinodal curve is the peaked curve (red) whose maximum is tangent to the third isobaric curve, and the negative slope of the lines of constant $P$ under the spinodal curve is an unstable region, where the heat capacity is negative. The temperature of the large-small black hole phase transition can in principle be obtained from the Maxwell equal area rule: the Gibbs free energy, at constant $J$,

$$
G(T, P, J)=M(S, P, J)-T S
$$

should be the same in both phases, $G_{l}=G_{s}$.

At constant $P$

$$
d G=-S d T+V d P=-S d T,
$$

so

$$
\begin{aligned}
0=G_{l}-G_{s}=\int_{S_{s}}^{S_{l}} d G & =-\int_{S_{s}}^{S_{l}} S d T=-[S T]_{s}^{l}+\int_{S_{s}}^{S_{l}} T(S) d S \\
\Leftrightarrow \int_{S_{s}}^{S_{l}} T(S) d S-\left(S_{l}-S_{s}\right) T & =0 .
\end{aligned}
$$


$\tilde{\mathrm{t}}$

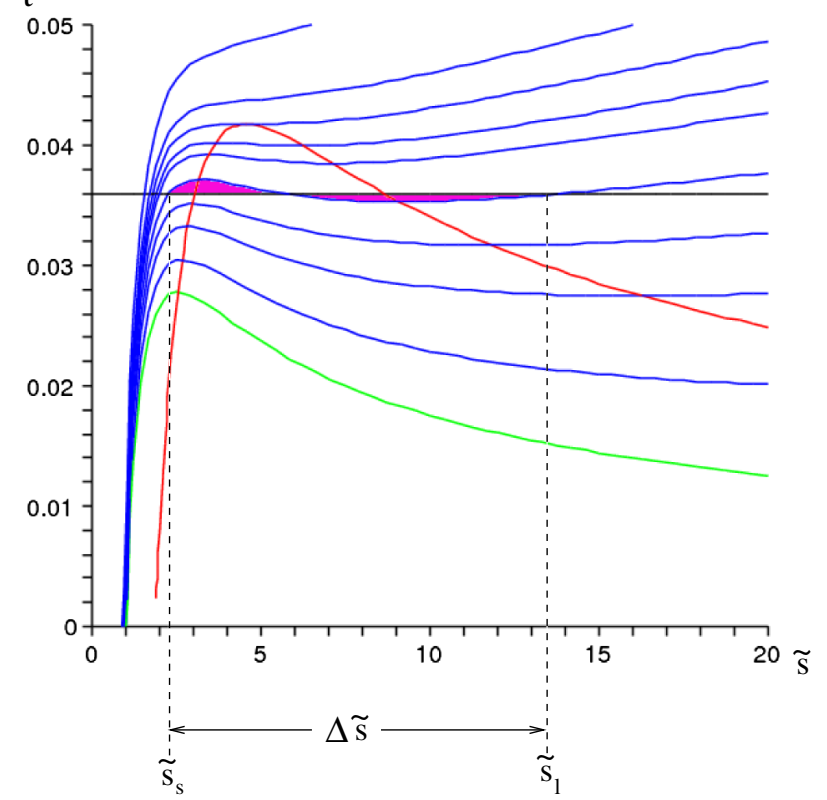

FIG. 4 (color online). Curves of constant $\tilde{p}$ in the $\tilde{s}-\tilde{t}$ plane. The lowest curve (green) is $\tilde{p}=0$. The the peaked curve (red) whose maximum is tangent to the third isobaric curve is the spinodal curve. The temperature of the phase transition is calculated using the Maxwell equal area rule.

The last expression determines the transition temperature, $T=T\left(S_{l}\right)=T\left(S_{S}\right)$, by demanding that the filled area in Fig. 4 be zero. The two phases coexist on a line in the $T-S$ plane, terminating at the critical point where the two sizes are equal (the Maxwell equal area rule for the first order Hawking-Page transition, associated with static AdSSchwarzschild, was investigated in the $P-V$ plane in [19]).

One must however be careful about applying ordinary thermodynamic intuition to black holes. In the $S-T$ plane the segment of an isotherm on which $T$ decreases with $S$ corresponds to negative heat capacity and signals an instability. The Maxwell rule states that, for the liquidgas phase transition, such an isotherm should be replaced by one which is horizontal between the two extremes of pure gas and pure liquid, the horizontal section of the isotherm being a mixture of liquid and gas in linear proportion to the distance to its two end points [16] (the argument is usually given in the $P-V$ plane, when compressibility replaces heat capacity, but it is essentially the same). There does not appear to be any such interpretation for black holes: a classical black hole solution is not a combination of large and small black holes, it is either one or the other and there does not seem to be any simple way in which the horizontal section of the constant $P$ curve in Fig. 4 can be thought of as a "mixture" of black holes with different entropies, as there is only one black hole. The negative heat capacity in the unstable region of Fig. 4, and the negative compressibility [20], may be a feature that one must live with, just like the negative heat capacity of asymptotically flat Schwarzschild black holes.

Based on the Clapeyron equation $\frac{d P}{d T}=\frac{\Delta S}{\Delta V}$, it follows from standard reasoning that the Gibbs free energy of the two phases agrees across the coexistence curve, $G_{l}(T, P)=G_{s}(T, P)$, but an explicit verification for the CCK transition would require solving a high order polynomial by numerical computation.

\section{CONCLUSIONS}

Phase transitions for asymptotically AdS-Kerr black holes have been analyzed in thermodynamic variables, viewing the (negative) cosmological constant as a pressure and taking its thermodynamically conjugate variable to be a volume. For nonrotating black holes the analytic form of the Hawking-Page transition line in the $P-T$ plane is quadratic (11) and the latent heat is inversely proportional to the temperature. At constant $\Omega$ the transition line is given parametrically in Eqs. (25) and (26) while the latent heat is (27) and is proportional to the mass per unit entropy. The Clapeyron equation has been explicitly checked to hold true, which is a consistency check on the thermodynamic interpretation of $\Lambda$ presented here. Analytic expressions are harder to obtain for constant $J$, but similar concepts apply.

From the AdS/CFT perspective the constant $\Omega$ case is relevant for rotating superfluids in the boundary conformal field theory. The main difference between the phase diagrams when $\Omega=0$ and $\Omega \neq 0$ is that the lower boundary of the black hole phase is at $P=0$ in the former case, and cannot be crossed for positive $P$, whereas it is at $P>0$ in the latter case, corresponding to Bose condensation on the boundary conformal field theory [11].

The phase diagram in higher dimensions is more complicated, due to the presence of more than one angular momentum, but is all the richer for that: the constant $J$ case exhibiting triple points [21] and reentrant phase transitions [22]. It would be interesting to explore the constant $\Omega$ phase diagram in higher dimensions in more detail. Bose condensation of vortices at a critical angular velocity for example could be a new phase when $\Omega \neq 0$.

There is much still to discover in this new picture of black hole thermodynamics.

\section{ACKNOWLEDGMENTS}

This research was supported in part by Perimeter Institute for Theoretical Physics. Research at Perimeter Institute is supported by the Government of Canada through Industry Canada and by the Province of Ontario through the Ministry of Economic Development and Innovation. 
[1] D. Kastor, S. Ray, and J. Traschen, Classical Quantum Gravity 26, 195011 (2009).

[2] B.P. Dolan, Classical Quantum Gravity 28, 125020 (2011).

[3] M. Cvetic, G. W. Gibbons, D. Kubizňák, and C. N. Pope, Phys. Rev. D 84, 024037 (2011).

[4] M. Henneaux and C. Teitelboim, Phys. Lett. 143B, 415 (1984); Phys. Lett. B 222, 195 (1989).

[5] C. Teitelboim, Phys. Lett. 158B, 293 (1985).

[6] M. M. Caldarelli, C. Cognola, and D. Klemm, Classical Quantum Gravity 17, 399 (2000).

[7] S. Gunasekaran, D. Kubizňák, and R. B. Mann, J. High Energy Phys. 11 (2012) 110.

[8] B. P. Dolan, in Open Questions in Cosmology, edited by G. J. Olmo, (InTech, 2012).

[9] R. Banerjee, S. K. Modak, and S. Samanta, Phys. Rev. D 84, 064024 (2011).

[10] R. Banerjee, S. K. Modak, and D. Roychowdhury, J. High Energy Phys. 10 (2012) 125.

[11] S. W. Hawking, C. J. Hunter, and M. M. Taylor-Robinson, Phys. Rev. D 59, 064005 (1999).
[12] Y. Brihaye and B. Hartmann, J. High Energy Phys. 09 (2010) 002.

[13] B. P. Dolan, Classical Quantum Gravity 28, 235017 (2011).

[14] S. W. Hawking and D. N. Page, Commun. Math. Phys. 87, 577 (1983).

[15] N. Altamirano, D. Kubizňák, R. B. Mann, and Z. Sherkatghanad, Galaxies 2, 89 (2014).

[16] H. B. Callen, Thermodynamics and an Introduction to Thermostatistics (Wiley, New York, 2006).

[17] G. W. Gibbons, M. J. Perry, and C. N. Pope, Classical Quantum Gravity 22, 1503 (2005).

[18] V. P. Maslov, Mathematical notes of the Academy of Sciences of the USSR 76, 697 (2004).

[19] E. Spallucci and A. Smailagic, J. Grav. 2013, 525696 (2013); Phys. Lett. B 723, 436 (2013).

[20] B. P. Dolan, Phys. Rev. D 84, 127503 (2011).

[21] N. Altamirano, D. Kubizňák, R. B. Mann, and Z. Sherkatghanad, Classical Quantum Gravity 31, 042001 (2014).

[22] N. Altamirano, D. Kubizňák, and R. B. Mann, Phys. Rev. D 88, 101502 (2013). 